\title{
X-ray grating interferometry with a liquid-metal-jet source
}

\author{
T. Thüring, ${ }^{1,2}$ T. Zhou, ${ }^{3}$ U. Lundström, ${ }^{3}$ A. Burvall, ${ }^{3}$ S. Rutishauser, ${ }^{1,2}$ C. David, ${ }^{1}$ \\ H. M. Hertz, ${ }^{3}$ and M. Stampanoni ${ }^{1,2}$ \\ ${ }^{1}$ Paul Scherrer Institute, Villigen PSI, Switzerland \\ ${ }^{2}$ Institute for Biomedical Engineering, Swiss Federal Institute of Technology, Zurich, Switzerland \\ ${ }^{3}$ Biomedical and X-Ray Physics, KTH Royal Institute of Technology, Albanova, SE-10691 Stockholm, Sweden
}

(Received 4 June 2013; accepted 16 August 2013; published online 27 August 2013)

\begin{abstract}
A liquid-metal-jet X-ray tube is used in an X-ray phase-contrast microscope based on a Talbot type grating interferometer. With a focal spot size in the range of a few microns and a photon flux of $\sim 10^{12}$ photons $/ \mathrm{s} \times \mathrm{sr}$, the brightness of such a source is approximately one order of magnitude higher than for a conventional microfocus source. For comparison, a standard microfocus source was used with the same grating interferometer, showing significantly increased visibility for the liquid-metal-jet arrangement. Together with the increased flux, this results in improved signalto-noise ratio. @ 2013 AIP Publishing LLC. [http://dx.doi.org/10.1063/1.4819766]
\end{abstract}

$\mathrm{X}$-ray microscopic computed tomography (micro CT) is nowadays a widely available technique for non-destructive examination of small samples in 3D. Resolutions down to a few microns, or even in the sub-micrometer range, are possible due to microfocus X-ray sources. In addition to high resolution, X-ray tubes with micrometer sized focal spots provide an X-ray beam of high spatial coherence, extending the range of applicable imaging techniques. High spatial coherence is required by most of the X-ray phase-contrast methods, with propagation-based phase-contrast imaging as a prominent example. ${ }^{1}$ Recent advances in X-ray phase-contrast imaging have demonstrated the use of a grating interferometer for simultaneous acquisition of absorption, phase, ${ }^{2,3}$ and dark field ${ }^{4}$ images. This technique has been applied with low-brilliance X-ray tubes ${ }^{5}$ and by using microfocus sources with a long interferometer arrangement ${ }^{6}$ as well as in a compact arrangement with typical micro CT settings. ${ }^{7}$

The small focal-spot size of microfocus sources, enabling high-resolution imaging, comes at the cost of a low output power and thus a low photon flux. The fundamental limitation of the flux in X-ray tubes is the maximum power load (power per target area) of the target. Since $99 \%$ of the kinetic energy of the electrons reaching the target is converted to heat, the maximum power load is determined by the maximum heat load, which is in turn limited by the melting point and the heat conductivity of the target material. The maximum output power of current microfocus sources with standard target materials (W, Mo) is typically $0.4-0.8 \mathrm{~W} / \mu \mathrm{m}$ diameter of the focal spot. ${ }^{8}$ The flux at this power level is normally on the order of $10^{11}$ photons $/ \mathrm{s} \times \mathrm{sr}$.

A recently developed X-ray tube based on a liquidmetal-jet anode is very promising as it overcomes this fundamental limit and provides a photon flux approximately one order of magnitude higher than for conventional microfocus sources. ${ }^{9,10}$ As opposed to a solid target material, these tubes operate with a liquid metal, which is typically a $\mathrm{Ga} / \mathrm{In} / \mathrm{Sn}$ (Galinstan) alloy, constantly circulating and formed into a well-shaped, thin jet at the position where the electron beam hits the liquid target. The temperature of the metal can be controlled in the circulation system and is kept at a temperature where it remains liquid. With a jet speed of around
$80 \mathrm{~m} / \mathrm{s}$, the highly regenerative target allows efficient heat dissipation and significantly increased power load compared to a solid target. Typical numbers of the current sources are around $10 \mathrm{~W}$ tube power per micrometer diameter of the focal spot, which is equivalent to an increase in brightness by around one order of magnitude compared to standard tubes.

The increased brightness (higher flux, smaller spot size) can significantly improve the performance of grating interferometer based micro CT systems, as it simultaneously provides better photon statistics and higher spatial coherence. Here, the potential gain of a liquid-metal-jet source for grating interferometry is investigated by evaluating the signalto-noise ratio (SNR) of a phase-contrast image of a phantom acquired with such a source and with a standard microfocus tube.

Fig. 1 shows a simulation of the spectra for a tungsten target with $4 \mathrm{~W}$ power and a liquid Galinstan target with $40 \mathrm{~W}$ power. The plot also shows the spectra if filtering is

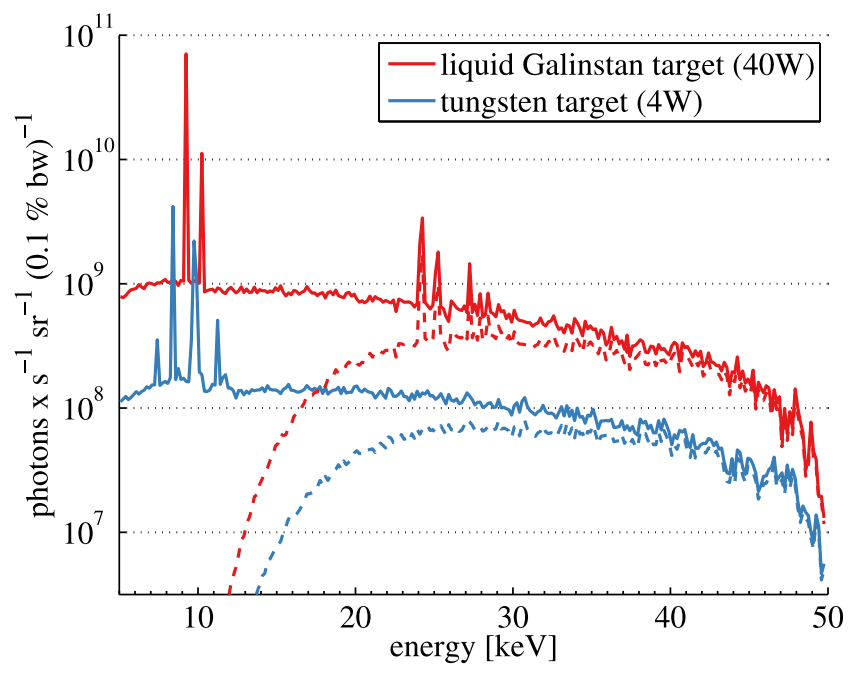

FIG. 1. Simulated spectra of a Galinstan $(\mathrm{Ga} / \mathrm{In} / \mathrm{Sn})$ target at $40 \mathrm{~W}$ and of a tungsten $(\mathrm{W})$ target at $4 \mathrm{~W}$, operated at a voltage of $50 \mathrm{kV}$. The total increase in flux of the Galinstan spectrum is 7.3. In addition, the dashed lines show the spectra if the beam is filtered with $18 \mathrm{~mm}$ of liquid paraffin (sample bath), $2 \mathrm{~mm}$ of PMMA (container), $50 \mu \mathrm{m}$ titanium (grating substrate), and $500 \mu \mathrm{m}$ of silicon (grating substrate). 
taken into account. According to these simulations, the mean increase of the flux over the entire energy range is 7.3. For the experiments presented here, the samples are scanned within a container filled with liquid paraffin to decrease the difference of the refractive indices between the sample and the surrounding. This slightly reduces the contrast at the border of the sample, but helps to avoid phase wrapping artifacts, which occur if the refraction angles become too large. On the other hand, it also acts as a filter in the beam which reduces the flux. In addition to the liquid paraffin bath, the grating substrates have to be considered as filters. For the filtered spectra, $18 \mathrm{~mm}$ of liquid paraffin, $2 \mathrm{~mm}$ of polymethylmethacrylate (PMMA) (container walls), $50 \mu \mathrm{m}$ titanium (beam splitter grating substrate), and $500 \mu \mathrm{m}$ of silicon (analyzer grating substrate) were taken into account.

Two experimental arrangements are involved in this study, each consisting of an X-ray tube, a grating interferometer, and a detector. Arrangement 1 is a standard arrangement with a conventional, solid tungsten target-based, microfocus source (Hamamatsu L10101). It is operated at a voltage of $50 \mathrm{kV}$ and a current of $80 \mu \mathrm{A}$, which corresponds to an output power of $4 \mathrm{~W}$ and a spot diameter of approximately $5 \mu \mathrm{m}$ in the direction orthogonal to the grating lines. The grating interferometer is a Talbot type interferometer (two gratings) with a beam splitter grating (phase grating) of period $p_{1}=4.12 \mu \mathrm{m}$ and an analyzer grating (absorption grating) of period $p_{2}=2.4 \mu \mathrm{m}$. The gratings were fabricated at Karlsruhe Institute of Technology (KIT). ${ }^{11}$ The design energy is $25 \mathrm{keV}$, at which the transmission function of the beam splitter introduces a periodic shift of zero and $\pi$ to the wave front. The Talbot order of the arrangement is $m=3$, yielding a source-to-detector distance of $1.05 \mathrm{~m}$ (source to analyzer grating). The detector is a CCD camera (Princeton Instruments) with a pixel size of $24 \mu \mathrm{m}$, a dynamic range of 16 bit, and a $\mathrm{Gd}_{2} \mathrm{O}_{2} \mathrm{~S}: \mathrm{Tb}$ (Gadox) scintillator $\left(20 \mathrm{mg} / \mathrm{cm}^{2}\right.$, $60 \mu \mathrm{m}$ thick) attached with a 1:1 fiber-optic coupling.

Arrangement 2 operates with a liquid-metal-jet X-ray tube with a Galinstan target. ${ }^{10}$ The electron beam is accelerated with a voltage of $50 \mathrm{kV}$ and a current of $0.8 \mathrm{~mA}$ (i.e., operating at $40 \mathrm{~W}$ ) and focused to a spot diameter of approximately $4 \mu \mathrm{m}$ in the direction orthogonal to the grating lines. The grating interferometer is identical to arrangement 1 (same gratings and same geometry) to achieve identical conditions. The detector is also a CCD camera (Photonic Science VHR), however, with a pixel size of $9 \mu \mathrm{m}$, a dynamic range of $12 \mathrm{bit}$, and a Gadox scintillator $\left(5 \mathrm{mg} / \mathrm{cm}^{2}\right.$, $15 \mu \mathrm{m}$ thick) with a 1:1 fiber-optic coupling.

The fact that the two systems have different X-ray cameras slightly complicates the comparison of the two experiments. To provide a fair comparison, the difference in SNR of the two detectors must be compensated by the exposure time and by binning. This compensation factor can be estimated by taking into account the pixel size $(a)$ and the detection quantum efficiency (DQE) of the cameras $(D)$. Using a $3 \times 3$ pixel binning for detector 2 , the pixel sizes are $a_{1}=24 \mu \mathrm{m}$ and $a_{2}=27 \mu \mathrm{m}$. According to the camera specifications, the DQEs for the simulated spectra (including filtering) are $D_{1}=0.249$ and $D_{2}=0.079$.

Neglecting the contribution of dark noise and readout noise in the detectors, the SNR is given by

$$
\mathrm{SNR} \propto \sqrt{t_{\exp } D a^{2}}
$$

To obtain an equivalent $\mathrm{SNR}$ of the detectors, $\mathrm{SNR}_{1}$ $=\mathrm{SNR}_{2}$, the compensation factor of the exposure time $t_{\mathrm{exp}}$ can then be calculated as

$$
\frac{t_{\mathrm{exp}, 1}}{t_{\mathrm{exp}, 2}}=\frac{D_{2}}{D_{1}} \frac{a_{2}^{2}}{a_{1}^{2}}=0.40 .
$$

This essentially means that the exposure time for detector 1 would have to be 2.5 times shorter than the exposure time of detector 2 to compensate for the SNR differences by the detectors.

Fig. 2 shows a slice in phase contrast of a phantom sample, acquired with a tomographic scan using the two arrangements. The outer wall of the phantom is PMMA and the circular rods are polyoxymethylene (POM), polypropylen (PP), and polystyrene (PS). As already discussed above, the sample has been scanned within a container filled with liquid paraffin to avoid phase wrapping. The effective pixel size, taking into account the geometric magnification, is $20 \mu \mathrm{m}$. The exposure time for arrangement 1 was $20 \mathrm{~s}$ per phase step and $1 \mathrm{~min}$ per phase step for arrangement 2 . The factor

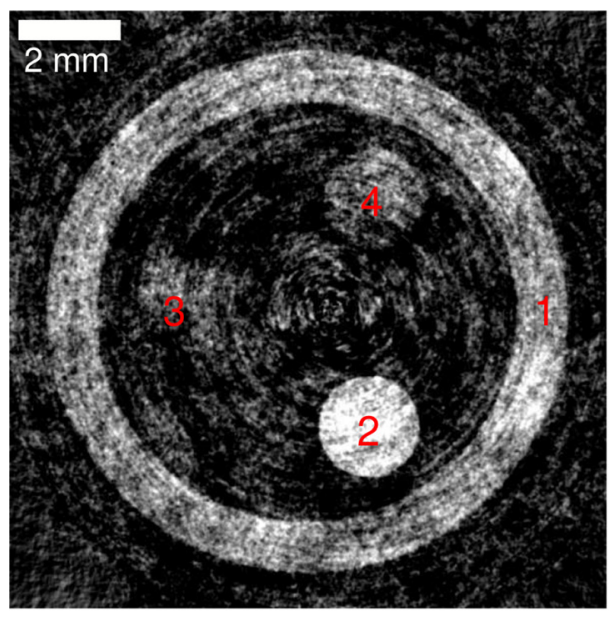

(a)



(b)
FIG. 2. Tomographic slice in phase contrast of a phantom acquired (a) with arrangement 1 (standard microfocus source) and (b) with arrangement 2 (liquid-metal-jet source). The materials of the phantom are PMMA (1), POM (2), PP (3), and PS (4). The background material is liquid paraffin, which was used to avoid phase wrapping artifacts. The SNR values for the different ROIs are listed in Table I. The grayscale is linear with black as the mean of the background (paraffin) and white as the mean of ROI 2 . 
TABLE I. SNR values of the phantom in the different ROIs, marked in the images of Fig. 2. The SNR was calculated by dividing mean and standard deviation from 100 tomographic slices and by averaging the values over an area inside the ROI

\begin{tabular}{lcrc}
\hline \hline & Arrangement 1 & Arrangement 2 & Ratio \\
\hline ROI 1 & $2.65( \pm 0.25)$ & $10.03( \pm 0.71)$ & $3.78( \pm 0.45)$ \\
ROI 2 & $4.67( \pm 0.51)$ & $18.30( \pm 2.20)$ & $3.91( \pm 0.66)$ \\
ROI 3 & $0.57( \pm 0.14)$ & $1.65( \pm 0.20)$ & $2.89( \pm 0.79)$ \\
ROI 4 & $1.22( \pm 0.18)$ & $4.55( \pm 0.41)$ & $3.73( \pm 0.70)$ \\
\hline \hline
\end{tabular}

between the exposure times closely corresponds to the theoretical factor of Eq. (2) that compensates for the efficiency difference of the camera systems in the two arrangements. The number of projections is 720 , and 5 phase steps have been acquired for each projection. The visibility ${ }^{12}$ is higher in arrangement $2\left(V_{1}=7.9 \%\right.$ and $\left.V_{2}=14.1 \%\right)$, which is mainly due to the smaller spot size of the liquid-metal-jet source. Taking into account visibility and flux and using $\mathrm{SNR} \propto \mathrm{V} \sqrt{\mathrm{f}},{ }^{13}$ the expected increase in SNR is

$$
\frac{\mathrm{SNR}_{2}}{\mathrm{SNR}_{1}}=\frac{V_{2}}{V_{1}} \sqrt{\frac{f_{2}}{f_{1}}}=4.8,
$$

where $f_{2} / f_{1}=7.3$ is the flux increase which was calculated from the simulated spectra. The measured SNR for each ROI and for both scans as well as the ratios are displayed in

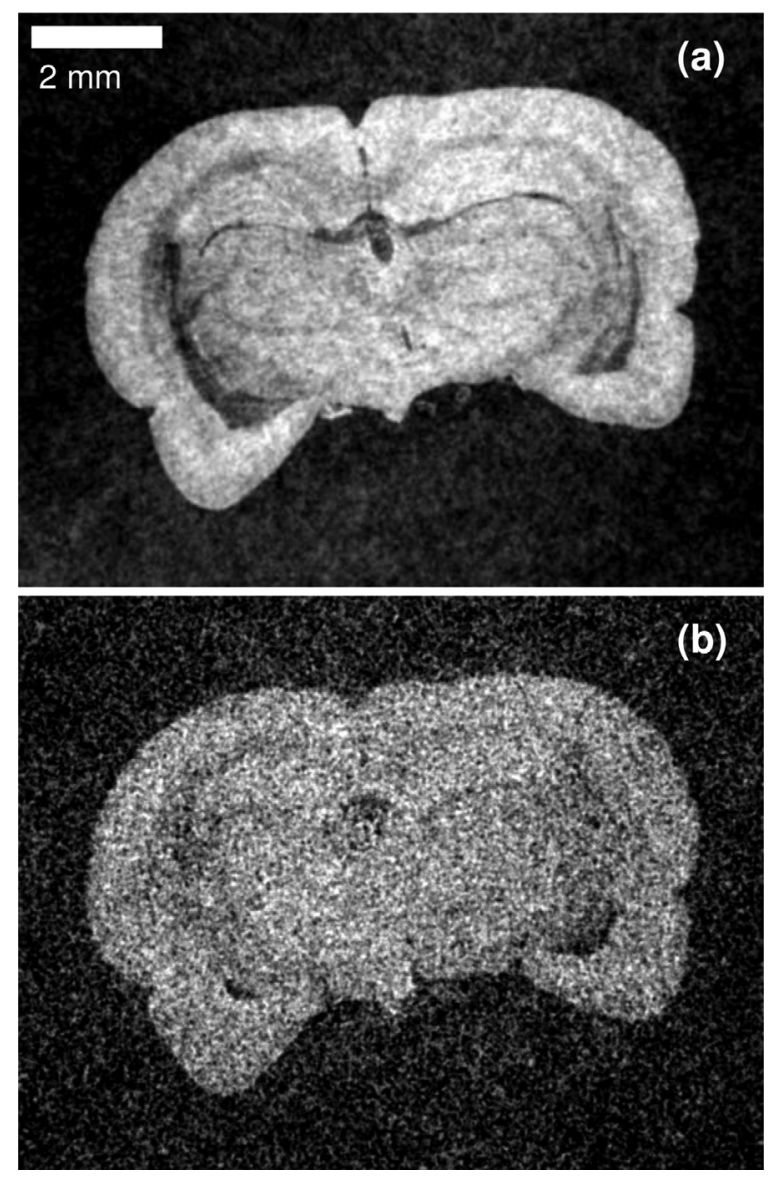

FIG. 3. Tomographic slice of a rat brain, in (a) phase contrast and (b) absorption contrast, obtained using arrangement 2 . The brain is fixed in paraffin and scanned in a container filled with liquid paraffin for the refractive index matching. The phase image reveals more details of the cortex.
Table I. With a mean ratio (all ROIs) of 3.6, the increase in SNR from arrangement 1 to arrangement 2 is a bit lower than theoretically expected. Possible reasons for this are uncertainties in the calculated spectra or the detector efficiencies.

To demonstrate a possible application of such a microscope, a tomography of a rat brain was recorded with arrangement 2 (liquid-jet source). The sample is fixed in paraffin and was scanned in the liquid paraffin bath. The geometry and the effective pixel size $(\sim 20 \mu \mathrm{m})$ are the same as for the phantom scan. The exposure time was 1 min per phase step. Fig. 3 shows a tomographic slice in phase and absorption contrast, showing a significant gain in contrast in the phase image.

In summary, X-ray grating interferometry has been demonstrated with a liquid-metal-jet X-ray source. In particular, such a source has great properties for microscopic imaging due to the increased brightness of the beam compared to conventional microfocus sources. For the source in this experiment, the flux was around 4 to 7 times higher, and the source size about $20 \%$ smaller, resulting in an increase of brightness by a factor of about 6 to 9 . The increased flux either yields reduced scan times or an increased SNR.

The exposure times at the resolution level used for these experiments are still rather long. Pushing the brightness of $\mathrm{X}$-ray sources even further with the innovative technology of liquid-metal targets may enable further improvements in exposure time and image quality in the future.

We thank Gordan Mikuljan from PSI, Switzerland, for this great support on mechanical construction issues. We also thank Daniel Larsson from KTH, Sweden, for discussions and help in preparing the experiments. We further thank Per Takman, Björn Hansson, and Oscar Hemberg from Excillum AB, Sweden, for providing relevant data. Karlsruhe Institute of Technology is acknowledged for providing the gratings. Finally, we acknowledge the Swedish Research Council, the Wallenberg Foundation, and the Göran Gustafsson foundation for financial support.

${ }^{1}$ S. Wilkins, T. Gureyev, D. Gao, A. Pogany, and A. Stevenson, Nature 384, 335 (1996).

${ }^{2}$ C. David, B. Nöhammer, H. Solak, and E. Ziegler, Appl. Phys. Lett. 81, 3287 (2002).

${ }^{3}$ A. Momose, S. Kawamoto, I. Koyama, Y. Hamaishi, K. Takai, and Y. Suzuki, Jpn. J. Appl. Phys., Part 2 42, L866 (2003).

${ }^{4}$ F. Pfeiffer, M. Bech, O. Bunk, P. Kraft, E. Eikenberry, C. Brönnimann, C. Grünzweig, and C. David, Nature Mater. 7, 134 (2008).

${ }^{5}$ F. Pfeiffer, T. Weitkamp, O. Bunk, and C. David, Nat. Phys. 2, 258 (2006).

${ }^{6}$ M. Engelhardt, J. Baumann, M. Schuster, C. Kottler, F. Pfeiffer, O. Bunk, and C. David, Appl. Phys. Lett. 90, 224101 (2007).

${ }^{7}$ T. Thüring, P. Modregger, T. Grund, J. Kenntner, C. David, and M. Stampanoni, Appl. Phys. Lett. 99, 041111 (2011).

${ }^{8}$ D. Grider, A. Wright, and P. Ausburn, J. Phys. D: Appl. Phys. 19, 2281 (1986).

${ }^{9}$ O. Hemberg, M. Otendal, and H. M. Hertz, Appl. Phys. Lett. 83, 1483 (2003).

${ }^{10}$ D. Larsson, P. Takman, U. Lundström, A. Burvall, and H. Hertz, Rev. Sci. Instrum. 82, 123701 (2011).

${ }^{11}$ J. Kenntner, T. Grund, B. Matthis, M. Boerner, J. Mohr, T. Scherer, M. Walter, M. Willner, A. Tapfer, M. Bech, F. Pfeiffer, I. Zanette, and T. Weitkamp, Proc. SPIE 7804, 780408 (2010).

${ }^{12}$ T. Weitkamp, A. Diaz, C. David, F. Pfeiffer, M. Stampanoni, P. Cloetens, and E. Ziegler, Opt. Express 13, 6296 (2005).

${ }^{13}$ K. J. Engel, D. Geller, T. Köhler, G. Martens, S. Schusser, G. Vogtmeier, and E. Rössl, Nucl. Instrum. Methods Phys. Res. A 648, 202 (2011). 